\title{
Solar-insolation-induced changes in the coma morphology of comet 67P/Churyumov-Gerasimenko
} Optical monitoring with the Nordic Optical Telescope

\author{
B. Zaprudin ${ }^{1}$, H. J. Lehto ${ }^{1}$, K. Nilsson ${ }^{2}$, A. Somero ${ }^{1}$, T. Pursimo ${ }^{3}$, C. Snodgrass ${ }^{4}$, and R. Schulz ${ }^{5}$ \\ 1 Tuorla Observatory, Department of Physics and Astronomy, University of Turku, Väisäläntie 20, 21500 Piikkiö, Finland \\ e-mail: bozapr@utu.fi \\ 2 Finnish Centre for Astronomy with ESO (FINCA), Väisäläntie 20, 21500 Piikkiö, Finland \\ 3 Nordic Optical Telescope, Apartado 474, 38700 Santa Cruz de La Palma, Spain \\ ${ }^{4}$ Planetary and Space Sciences, School of Physical Sciences, The Open University, Milton Keynes, MK7 6AA, UK \\ 5 ESA, Scientific Support Office, ESTEC, PO Box 299, 2200 AG Noordwijk, The Netherlands
}

Received 20 January 2017 / Accepted 5 May 2017

\begin{abstract}
Context. 67P/Churyumov-Gerasimenko (67P/C-G) is a short-period Jupiter family comet with an orbital period of $6.55 \mathrm{yr}$. Being the target comet of ESA's Rosetta mission, $67 \mathrm{P} / \mathrm{C}-\mathrm{G}$ has become one of the most intensively studied minor bodies of the solar system. The Rosetta Orbiter and the Philae Lander have brought us unique information about the structure and activity of the comet nucleus, as well as its activity along the orbit, composition of gas, and dust particles emitted into the coma. However, as Rosetta stayed in very close proximity to the cometary nucleus (less than $500 \mathrm{~km}$ with a few short excursions reaching up to $1500 \mathrm{~km}$ ), it could not see the global picture of a coma at the scales reachable by telescopic observations $\left(10^{3}-10^{5} \mathrm{~km}\right)$.

Aims. In this work we aim to connect in-situ observations made by Rosetta with the morphological evolution of the coma structures monitored by the ground-based observations. In particular, we concentrate on causal relationships between the coma morphology and evolution observed with the Nordic Optical Telescope (NOT) in the Canary Islands, and the seasonal changes of the insolation and the activity of the comet observed by the Rosetta instruments.

Methods. Comet 67P/C-G was monitored with the NOT in imaging mode in two colors. Imaging optical observations were performed roughly on a weekly basis, which provides good coverage of short- and long-term variability. With the three dimensional modeling of the coma produced by active regions on the southern hemisphere, we aim to qualify the observed morphology by connecting it to the activity observed by Rosetta.

Results. During our monitoring program, we detected major changes in the coma morphology of comet $67 \mathrm{P} / \mathrm{C}-\mathrm{G}$. These were long-term and long-lasting changes. They do not represent any sudden outburst or short transient event, but are connected to seasonal changes of the surface insolation and the emergence of new active regions on the irregular shaped comet nucleus. We have also found significant deviations in morphological changes from the prediction models based on previous apparitions of 67P/C-G, like the time delay of the morphology changes and the reduced activity in the northern hemisphere. According to our modeling of coma structures and geometry of observations, the changes are clearly connected with the activity in the southern hemisphere observed by the Rosetta spacecraft.
\end{abstract}

Key words. comets: individual: 67P/C-G

\section{Introduction}

Whilst comet 67P/Churyumov-Gerasimenko (hereafter 67P/CG) was under continuous investigation by the scientific instruments on board the Rosetta spacecraft for over two years (since in August 2014), relatively little has been done yet to connect spacecraft data with the remote-sensing observations from the ground. Rosetta provides us with a vast amount of precious information about one comet, its target 67P/C-G, but does not give us information about any other comets in the solar system. However, we can visit a comet only once in a while, hence we must rely on ground-based observations when gathering information about the ensemble of comets. Therefore, it is of utmost importance that for $67 \mathrm{P} / \mathrm{C}-\mathrm{G}$ the spacecraft measurements are linked with observations from the ground in order to better understand and interpret the results of ground-based observations. In this manner, we will eventually be able to transfer what we learn from Rosetta to other comets only observed from the ground.

The Rosetta mission has provided us with the unique opportunity to monitor the evolution of comet $67 \mathrm{P} / \mathrm{C}-\mathrm{G}$ along its orbit around the Sun from close proximity and in parallel with observations from ground-based telescopes. In this work, we present our observations with the Nordic Optical Telescope (NOT) on the detailed evolution of 67P/C-G coma morphology, compare it with the predictions based on past observations, and connect it to the nucleus surface illumination and local activity data obtained with the Rosetta spacecraft.

Changes in the global morphology of the coma observed from the ground can be connected with the seasonal changes in insolation of the surface of the comet nucleus as identified by Rosetta. The first evidence of a similar connection was suggested by Sekanina in 1988 to explain periodic changes in the coma 


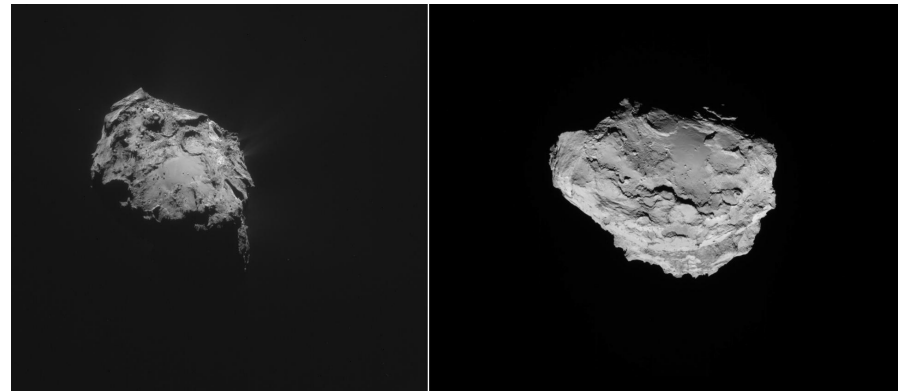

Fig. 1. Rosetta NavCam images of comet $67 \mathrm{P} / \mathrm{C}-\mathrm{G}$ from roughly the same perspective taken in November 2015 (left), comet's austral summer, and in August 2014 (right), austral winter. The illumination of different areas of the comet nucleus surface is clearly seen in those two images.

structure of comet 2P/Encke (Sekanina 1988). For comet 67P/C$\mathrm{G}$ seasonal effects on coma morphology were already predicted before the arrival of the spacecraft (Vincent et al. 2013) and were later confirmed by the in-situ measurements obtained with Rosetta. In May 2015 new areas of the comet nucleus were exposed to the sunlight, starting summer season on the southern hemisphere (Tosi et al. 2016). This can also be confirmed by the Rosetta NavCam Public domain images (Fig. 1) and by the series of outbursts observed by the OSIRIS camera on board Rosetta (Vincent et al. 2016). In the subsequent time period, the morphology of the coma as observed from the ground also started to evolve from an almost symmetrical profile, with a visible dust tail and a subsolar fan into a highly asymmetrical structure with two jets, one emerging into roughly subsolar direction and the other roughly in the southern direction in the coma as projected on the plane of the sky. A similar structure was already detected during the post-perihelion phase of the apparition in 2003 (Weiler et al. 2004), but not in the pre-perihelion observations in 2008 (Levasseur-Regourd et al. 2010). This indicates that the observed change had returned during the post-perihelion phase of the comet for at least two apparitions, hence is periodic and connected with the averaged activity over a certain orbital phase - in other words with the average seasonal activity.

Our observations were obtained under exceptional geometrical conditions, that is, the Earth moved in the same direction as the comet along its orbit. Therefore ground-based observers had the unique opportunity to observe the target from almost the same geocentric distance for a prolonged period of time, covering a wide range of heliocentric distances and seasonal changes. While the heliocentric distance of the comet, $r$, changed continuously, the spatial scale of the images remained almost constant, which made it possible to compare the observed coma features without having to deal with effects of changing spatial resolution in the images. Although a large variety of telescopes were observing comet 67P/C-G in support of the Rosetta mission (Snodgrass et al. 2017), the majority of those observations were not continuous and do not cover all periods of activity evolution. The observations with the NOT have the advantage that the comet was regularly observed over almost the entire period of its visibility from the ground with the same instrument configuration and even with almost the same image scale. While other monitoring programs such as that described in Boehnhardt et al. (2016) focused mostly on the photometry and estimation of dust parameters, our work concentrates on the analysis of the temporal evolution of the coma morphology and aims at providing a qualitative explanation for the observed phenomena.

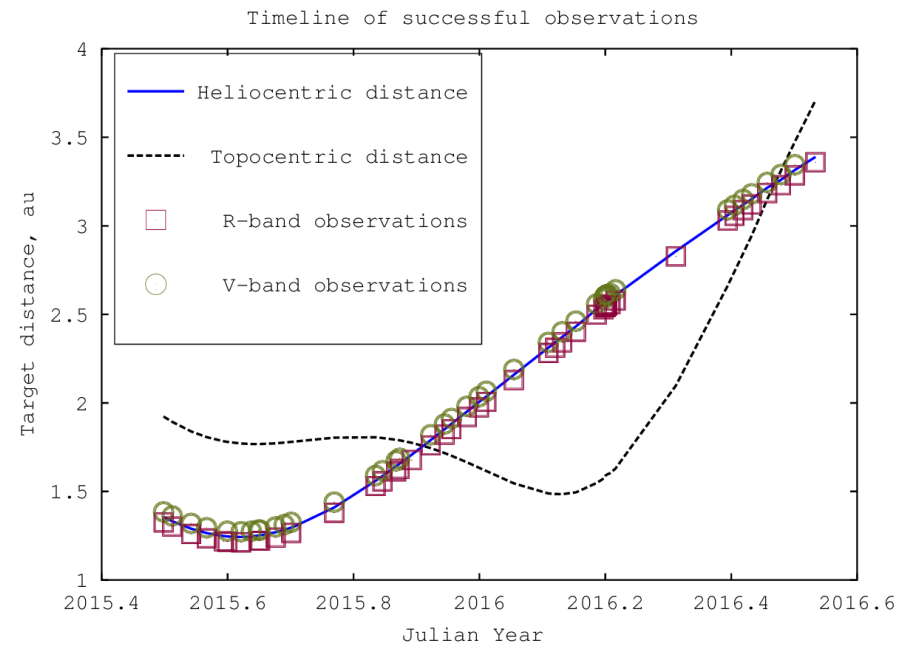

Fig. 2. Heliocentric and topocentric distances of the comet $67 \mathrm{P} / \mathrm{C}-\mathrm{G}$ as a function of time. Successful observations performed with the NOT are marked with squares or circles.

\section{Observations}

Our observations were performed with the NOT - a 2.56-m telescope located on La Palma, Canary Islands, Spain, using the ALFOSC or the StanCam (backup) instrument in the imaging mode with standard Bessel $V$ and $R$ filters. ALFOSC is an optical imaging and spectroscopy instrument with a field of view of $6.4^{\prime} \times 6.4^{\prime}$ and angular resolution of $0.189^{\prime \prime}$ before 31 March 2016 or $0.213^{\prime \prime}$ after 31 of March 2016 due to replacement of the CCD matrix. StanCam is a CCD-camera with a field of view of $3 \times 3$ arcmin and angular resolution of $0.179 \mathrm{arcsec}$. The typical seeing of the NOT is $0.45^{\prime \prime}-1^{\prime \prime}$, thus the true spatial resolution runs from $550 \mathrm{~km}$ to $1700 \mathrm{~km}$, here calculated for the heliocentric distance $r=1.7 \mathrm{AU}$, but approximately valid for most of the observations. The scales at which we observe are much larger than the scales of any Rosetta measurement and therefore physical features like jets cannot be directly compared.

A typical observation block had $6 \times 200 \mathrm{~s}$ exposures in the $V$ band and $7 \times 200 \mathrm{~s}$ exposures in the $R$ band in addition to the calibration frames. To enhance the signal-to noise-ratio $(\mathrm{S} / \mathrm{N})$, we added up all the images into a single one for each night and each filter, excluding images where the coma was badly contaminated with any background object like a star or a galaxy. The first successful observations of 67P/C-G after Solar conjunction were performed on 1 July 2015 in the early morning - almost at twilight. Thereafter, the target was monitored on a weekly basis, but some of the observations were of a reduced quality due to the poor atmospheric conditions or Moonlight. The timeline of the successful observations is shown in Fig. 2 and detailed information is presented in Tables A.1 and A.2.

\section{Analysis}

\subsection{Image reduction}

Images were reduced and calibrated using the standard procedures of bias level subtraction and flat-field correction with IRAF (Tody 1986). Background objects contaminating the coma were manually masked to avoid biasing of the results. This can be seen as dark stripes on the images in Fig. 4.

To reveal asymmetrical coma structures and enhance contrast on morphological details we have processed our images of comet $67 \mathrm{P} / \mathrm{C}-\mathrm{G}$ in the following way: the azimuthally averaged 
brightness was plotted as a function of distance from the optocenter, which is also the brightest point of the coma and the position of the comet nucleus. After a smoothing with the cubic spline method, the azimuthally averaged images were subtracted from the original ones. This way any deviation from the circularly symmetrical coma profile would be clearly visible.

The results were verified using two other techniques, unsharp masking and elliptical isophote fitting with no significant difference to our first method. This excludes artifacts as a possibility. Therefore we have continued with the simplest and the most straightforward method described above. Series of processed images covering main evolution steps of the coma are presented in Fig. 4. The directions of the jets as projected on the plane of the sky were measured as an angle between celestial north and the best fit point of maximum surface brightness associated with the jet at a projected distance of $10^{4} \mathrm{~km}$. The error limits provided are defined from the output of the fitting routine ( $3 \sigma$ limits) combined with the sensitivity of the results to the exact subpixel position of the optocenter. The results are presented in Tables A.1 and A.2.

For several epochs where both filters were used, a color analysis was performed. The observed images in $R$ band were shifted, normalized, and subtracted from the normalized $V$ band images of the same night. The resulting images appeared flat, apart from some artifacts caused by different seeing for the $R$ and $V$ filters, and no significant color differences in morphological features of the coma were observed within the available $S / N$. This may be explained by either a relatively uniform dust-to-gas ratio or a rather small contribution of the gaseous coma to the total brightness, which is caused by a high dust-to-gas ratio also reported by Moreno et al. (2016).

\subsection{Three-dimensional modeling of coma}

The images we obtain with the telescope are only twodimensional projections of real three-dimensional phenomena. Therefore, to provide an interpretation of observed morphological features, we have to construct a three-dimensional model, which in projection should reproduce our observations. With the Rosetta observations (Preusker et al. 2015) the rotational axis orientation and the rotation speed of the nucleus were measured with extremely high precision.

In the simplest case, the activity of the southern regions of the comet can be approximated as a single large-scale diffuse jet, which is ejecting dust and gas in the direction perpendicular to the imaginary spherical surface of a coordinate grid. The average propagation velocity of the dust can be estimated from the results of Boehnhardt et al. (2016), who determine a coma dust expansion velocity up to $150 \pm 30 \mathrm{~m} / \mathrm{s}$ at $r=1.25 \mathrm{AU}$. This means it would take less than a day for the dust to reach distances of $10^{4} \mathrm{~km}$. Therefore, the propagation time of the dust can be neglected in the model. As the rotation period is only $12 \mathrm{~h}$ (Preusker et al. 2015), the jet is smeared into a conical cloud of dust, with the symmetry axis parallel to the rotational axis of the comet and with an opening angle of $2 \times\left(90^{\circ}-\right.$ lat $)$, where lat is a latitude of the radial direction of the dust ejection. In a spherical model of a cometary nucleus, this number would also be the latitude of the location of the active area on the cometary surface. However, as the shape of the nucleus is irregular, the direction normal to the surface deviates significantly from one predicted solely by the topological latitude. As the coma is optically thin, we can integrate its density along the line of sight and reproduce the two-dimensional projection we observe from the ground. In that way, our model also resolves the continuously changing apparent geometry in our observations.

For this work, we assumed a latitude of the dust ejection direction around $55^{\circ}$ South, as it reproduces the morphology of the coma as observed from the ground in the later stages (see Fig. 3) and is in a plausible agreement with the Rosetta data (Vincent et al. 2016) and with the models for the previous apparitions of 67P/C-G (Vincent et al. 2013).

There are several important assumptions made in this model. First of all, the activity may be strongly modulated by the daynight circle on the comet, intensifying one side of the conical fan in comparison to the other. For instance, the small outbursts around perihelion were reported to show a regularity in the local time that indicates a connection with the rotation of the nucleus (Vincent et al. 2016). However, later in the postperihelion phase a significant night side activity was observed as well (Knollenberg et al. 2016). This may explain why the structures predicted by the model are in much better agreement with the observations beyond $r=1.5 \mathrm{AU}$ than closer to perihelion. In the future, as more of the Rosetta data on comet 67P/C-G's activity is published and it becomes possible to perform a deep meta-analysis, the location of the active regions could be traced separately for each epoch of our observations and we can investigate the timescales and causes of the evolution of morphological features in great detail.

\section{Results}

The morphology of the coma structure of comet $67 \mathrm{P} / \mathrm{C}-\mathrm{G}$ has been followed for the entire visibility period with frequent imaging with the NOT in $R$ and $V$ bands and the information on its evolution has been successfully obtained. Some of those images are presented in Fig. 4 to cover the critical steps of observed evolution of the coma. The details of the successful $R$-band and $V$-band observations are presented in Tables A.1 and A.2, respectively.

The morphology of the coma structure of comet $67 \mathrm{P} / \mathrm{C}-\mathrm{G}$ has gradually undergone significant changes after the perihelion passage (13 of August 2015). As seen in the upper left corner of Fig. 4, the pre-perihelion coma shows a major tail feature pointing in the direction of the orbital plane and a short $\left(\leq 5 \times 10^{3} \mathrm{~km}\right)$ strongly curved subsolar fan pointing roughly in the direction of the Sun. All asymmetries of the coma can be explained by the angle of incidence of the comet's orbital plane. A similar structure of the coma has also been observed before the Solar conjunction (Zaprudin et al. 2015; Snodgrass et al. 2017). After the perihelion passage the subsolar fan intensified compared with the tailward activity and its measured surface brightness profile changed. This is in good agreement with the series of outbursts and changes in the activity observed by the Rosetta spacecraft (Vincent et al. 2016). In late August 2015, roughly a week after the perihelion passage, the subsolar jet started to branch into two separate jets (see Fig. 4).

Although the new features appear in the southern hemisphere of the coma, our model fails to reproduce the observations if continuously active areas are assumed on the southern hemisphere of the nucleus, as shown in Fig. 3. The possible explanations for this may be the day-night cycle modulation discussed in Sect. 3.2 or the obscuration of the jet with the coma components caused by other activity.

Later, after their complete separation, the two jets were also observed by other telescopes (Snodgrass et al. 2017; Boehnhardt et al. 2016; Knight et al., in prep.). While the subsolar component was constantly pointing roughly in the projected 

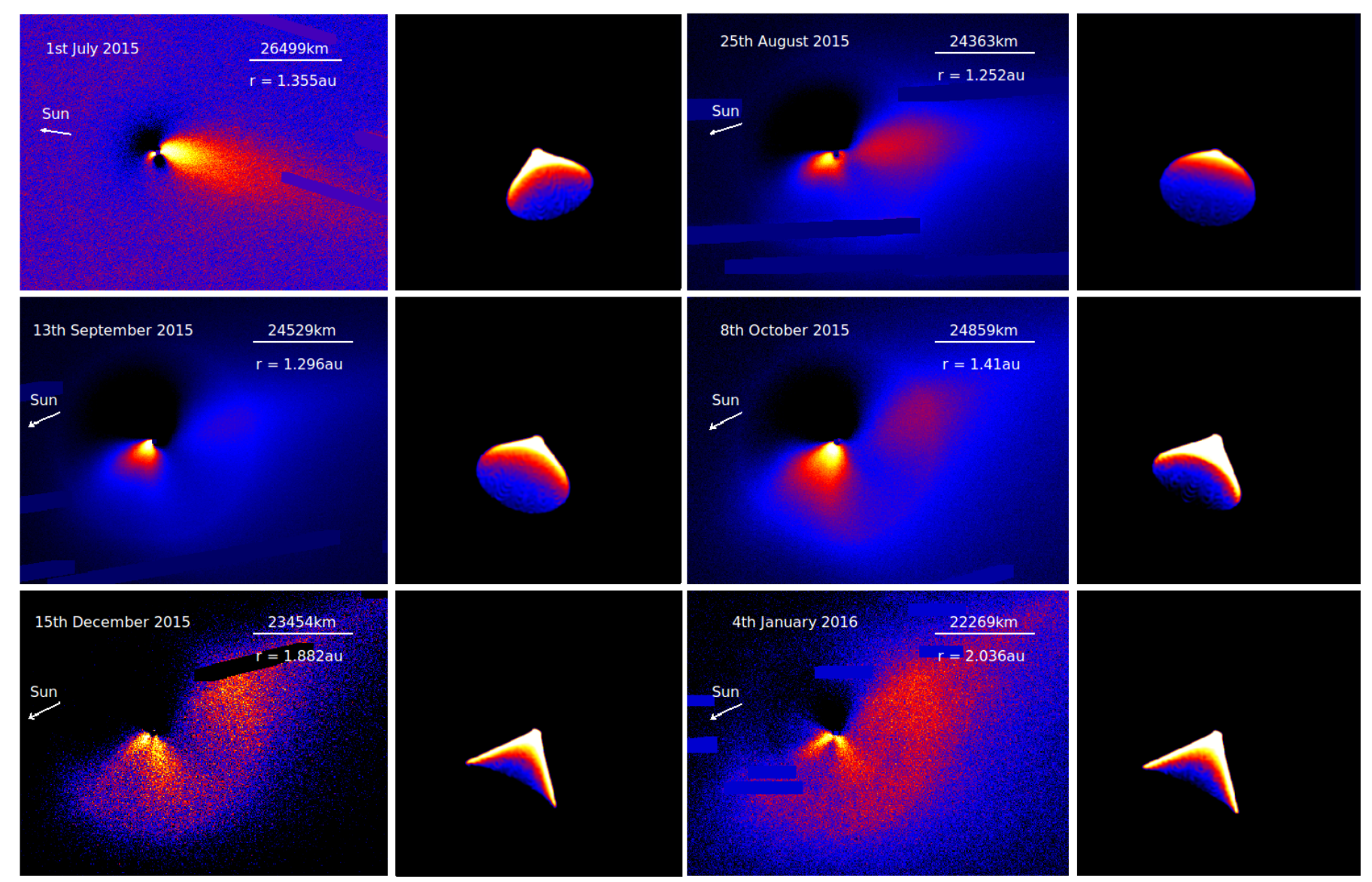

Fig. 3. Pairs of images of actual observations compared to the calculated southern fan model prediction for the same epoch. The model implies that the conical coma is caused by a single active area ejecting dust and gas in the radial direction with corresponding latitude $55^{\circ}$ South. The jets are modeled to be optically thin and to extend $20000 \mathrm{~km}$ into space. Model predictions for all the observations in spring 2016 are similar to one for January 2016 and are therefore not shown on the image.

direction of the Sun, the secondary southern jet slowly turned counter-clockwise until February-March 2016 (see Fig. 4). In our model, the two separate jets are simply the projected edges of the three-dimensional conical fan produced by a single active region on the rapidly rotating comet nucleus. The opening angle of the cone, however, is not in exact agreement with the topological latitudes $\left(0^{\circ}\right.$ to $40^{\circ}$ South) of the new active regions reported by Vincent et al. (2016). This may well be explained by the irregularity of the shape of the nucleus, where the surface normal vector direction deviates significantly from the one predicted by the topological latitude angle of a sphere. In future studies, a full three-dimensional surface model shall be used to calculate the direction of the dust plume propagation for each outburst or activity spot detected.

The morphological changes were also observed with a significant time delay compared with the predictions made from observations during past apparitions of $67 \mathrm{P} / \mathrm{C}-\mathrm{G}$ (Vincent et al. 2013). According to those models, the coma would already have developed separate jet structures at the time of perihelion. In addition to the subsolar fan, the secondary jet was expected to point southward and the tertiary jet to point in the northward direction. In contrast to this, we observed the appearance of the secondary southern jet only weeks later, pointing in a direction that would not be expected from the simple activity model and the observation geometry (see Fig. 3 for the September and October observations), and the northern jet was extremely faint or not detected at all in our observations, although we note that Vincent et al. (2013) used a different coma enhancement technique (Laplacian filter).

\section{Discussion}

The changes in the morphology of the coma can be explained by the seasonal variation of the illumination of the irregular nucleus surface. In particular, the southern hemisphere of the nucleus has been experiencing winter for more than five years staying far away from the Sun (Tosi et al. 2016). However, in May 2015 a short summer started and ignited whole new activity regions on the southern hemisphere. This was observed by the Rosetta instruments as the series of outbursts (Vincent et al. 2016), the emergence of new jets, and an overall increase in gas and dust production rates (Accomazzo et al. 2016).

In our data, those changes have appeared with a time delay with respect to the change in illumination conditions or with the activity observed by Rosetta (Vincent et al. 2016). This delay is significantly larger than the time required for the dust to move to a distance of about $10000 \mathrm{~km}$ from the nucleus - less than one day assuming the terminal velocities of the dust measured by Boehnhardt et al. (2016). Besides that, we must note that our simple model is insufficient if the activity of the southern regions is variable; for example if it is strongly modulated by a day-night circle or modified by other temporarily active regions. Extremely 
B. Zaprudin et al.: Solar-insolation-induced changes in the coma morphology of comet $67 \mathrm{P} /$ Churyumov-Gerasimenko

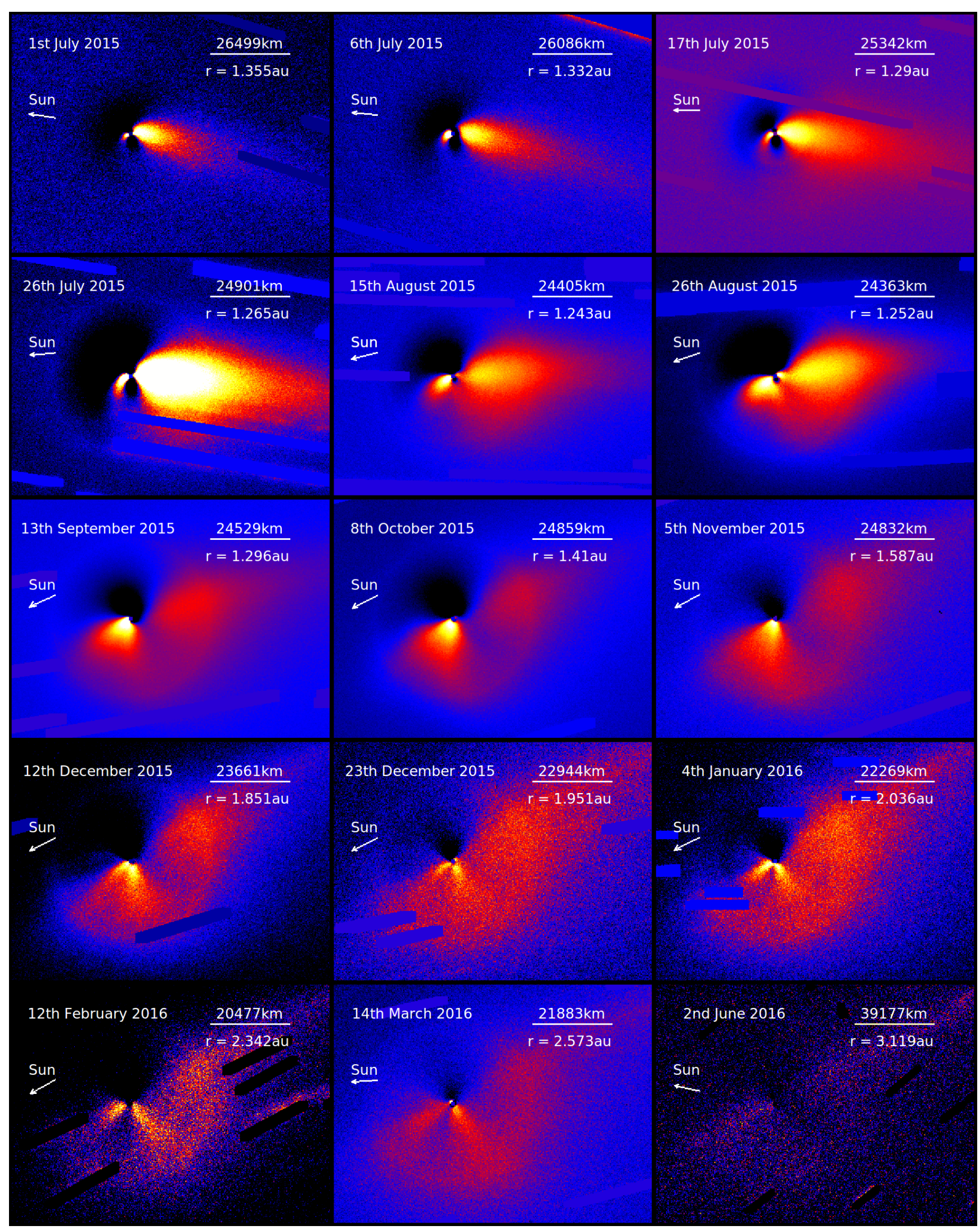

Fig. 4. Processed images of comet $67 \mathrm{P} / \mathrm{C}-\mathrm{G}$ observed in $R$-band with the NOT show main evolution steps of the coma morphology. All images have celestial north pointing up and the direction of the Sun indicated with an arrow. A heliocentric distance $r$ of the target and a spatial scale of the image at the moment of observation is presented in a right-hand corner of each image. The scale is given as the length of a 100-pixel-long white line projected to the distance of the target. Color scale is arbitrary and does not reflect the absolute brightness of the object. 
faint northern jets were not a complete surprise, as the comet has shown reduced activity on the northern hemisphere over several apparitions and a possible disappearance of the northern jet was already speculated by Vincent et al. (2013).

As we know from previous studies (Schulz et al. 2015), the properties of the dust particles evolve over the activity period of comet $67 \mathrm{P} / \mathrm{C}-\mathrm{G}$. Therefore, as the new fresh surfaces became active, it would not be surprising for the properties of the dust particles to also change. However, no significant changes in dust flux or size distribution were reported by the Rosetta dust analysis instrument COSIMA (Langevin et al. 2016; Merouane et al. 2016), which could however be due to either low statistics or the fact that ground-based dust observations focus on much smaller particles than those collected by the COSIMA instrument. In our data, we observe an extremely curved subsolar jet around the perihelion (see Fig. 4), which later appeared rather straight. This may partly be due to the effect of changing observational geometry (the target was in opposition in March 2016), but may also indicate that the subsolar jet, although projected on the same area of the image, might actually not be the same jet with respect to its origin on the nucleus. Further investigation of dust properties may require spectroscopic and polarization observations of the coma, such as those performed by Hadamcik et al. (2010) during the 2008-2009 apparition of 67P/C-G and several still unpublished observations made during the last apparition.

Acknowledgements. Work was supported by the Academy of Finland grant number 277375 (H.L., B.Z., A.S.). This research is based on observations made with the Nordic Optical Telescope, operated by the Nordic Optical Telescope Scientific Association at the Observatorio del Roque de los Muchachos, La Palma,
Spain, of the Instituto de Astrofisica de Canarias. The data presented here were obtained with ALFOSC, which is provided by the Instituto de Astrofisica de Andalucia (IAA) under a joint agreement with the University of Copenhagen and NOTSA.

\section{References}

Accomazzo, A., Ferri, P., Lodiot, S., et al. 2016, Acta Astron., 126, 190 Boehnhardt, H., Riffeser, A., Kluge, M., et al. 2016, MNRAS, 462, S376 Hadamcik, E., Sen, A. K., Levasseur-Regourd, A. C., Gupta, R., \& Lasue, J. 2010, A\&A, 517, A86

Knollenberg, J., Lin, Z. Y., Hviid, S. F., et al. 2016, A\&A, 596, A89

Langevin, Y., Hilchenbach, M., Ligier, N., et al. 2016, Icarus, 271, 76

Levasseur-Regourd, A.-C., Hadamcik, E., Sen, A. K., Gupta, R., \& Lasue, J. 2010, in Icy Bodies of the Solar System, eds. J. A. Fernandez, D. Lazzaro, D. Prialnik, \& R. Schulz, IAU Symp., 263, 259

Merouane, S., Zaprudin, B., Stenzel, O., et al. 2016, A\&A, 596, A87

Moreno, F., Snodgrass, C., Hainaut, O., et al. 2016, A\&A, 587, A155

Preusker, F., Scholten, F., Matz, K.-D., et al. 2015, A\&A, 583, A33

Schulz, R., Hilchenbach, M., Langevin, Y., et al. 2015, Nature, 518, 216

Sekanina, Z. 1988, AJ, 96, 1455

Snodgrass, C., A'Hearn, M. F., Aceituno, F., et al. 2017, Phil. Trans. R. Soc. A, 375,20160249

Tody, D. 1986, in Society of Instrumentation in astronomy VI, ed. D. L. Crawford, SPIE Conf. Ser., 627, 733

Tosi, F., Capaccioni, F., Filacchione, G., et al. 2016, in EGU General Assembly Conference Abstracts, 18, 17371

Vincent, J.-B., Lara, L. M., Tozzi, G. P., Lin, Z.-Y., \& Sierks, H. 2013, A\&A, 549, A121

Vincent, J.-B., A'Hearn, M. F., Lin, Z.-Y., et al. 2016, MNRAS, 462, S184

Weiler, M., Rauer, H., \& Helbert, J. 2004, A\&A, 414, 749

Zaprudin, B., Lehto, H. J., Nilsson, K., et al. 2015, A\&A, 583, A10 
B. Zaprudin et al.: Solar-insolation-induced changes in the coma morphology of comet 67P/Churyumov-Gerasimenko

\section{Appendix A: Additional tables}

Table A.1. Successful observations of comet 67P/C-G in $R$-band with the Nordic Optical Telescope.

\begin{tabular}{|c|c|c|c|c|c|c|c|c|c|}
\hline $\begin{array}{c}\text { Date of } \\
\text { observation }\end{array}$ & UT & Filter & $\begin{array}{c}\text { Exposure } \\
\text { time (s) }\end{array}$ & $r$ & Delta & $\begin{array}{l}\text { Phase } \\
\text { angle }\end{array}$ & $\begin{array}{l}\text { Direction of } \\
\text { the Sun (deg) }\end{array}$ & $\begin{array}{c}\text { Subsolar jet } \\
\text { direction (deg) }\end{array}$ & $\begin{array}{c}\text { southern jet } \\
\text { direction (deg) }\end{array}$ \\
\hline 20150701 & 05:00:28.8 & $R$ & 1400 & 1.355 & 1.923 & 30.5 & 278.4 & $198 \pm 5^{\circ}$ & Not visible \\
\hline 20150706 & 05:00:50.2 & $R$ & 1400 & 1.332 & 1.893 & 31.1 & 275.9 & $208 \pm 5^{\circ}$ & Not visible \\
\hline 20150717 & 04:44:09.4 & $R$ & 1400 & 1.290 & 1.839 & 32.4 & 270.1 & $216 \pm 3^{\circ}$ & Not visible \\
\hline 20150726 & $04: 37: 34.6$ & $R$ & 1400 & 1.265 & 1.807 & 33.1 & 265.3 & $220 \pm 3^{\circ}$ & Not visible \\
\hline 20150805 & $04: 38: 42.8$ & $R$ & 1400 & 1.248 & 1.783 & 33.6 & 260.1 & Contaminated & Not visible \\
\hline 20150807 & $04: 42: 37.8$ & $R$ & 1400 & 1.246 & 1.780 & 33.7 & 259.1 & $227 \pm 4^{\circ}$ & Not visible \\
\hline 20150815 & 04:39:09.7 & $R$ & 1400 & 1.243 & 1.771 & 33.9 & 255.2 & $232 \pm 3^{\circ}$ & 1678 \\
\hline 20150825 & $05: 01: 35.3$ & $R$ & 1400 & 1.252 & 1.768 & 34.0 & 250.8 & $233 \pm 3^{\circ}$ & $176 \pm 6^{\circ}$ \\
\hline 20150826 & 04:59:58.1 & $R$ & 1400 & 1.252 & 1.768 & 34.0 & 250.4 & $239 \pm 2^{\circ}$ & $173 \pm 4^{\circ}$ \\
\hline 20150904 & $05: 25: 45.1$ & $R$ & 1400 & 1.270 & 1.772 & 33.9 & 247.0 & $236 \pm 3^{\circ}$ & $190 \pm 5^{\circ}$ \\
\hline 20150913 & $04: 52: 50.1$ & $R$ & 1400 & 1.296 & 1.780 & 33.8 & 244.2 & $237 \pm 3^{\circ}$ & $189 \pm 5^{\circ}$ \\
\hline 20151008 & $05: 22: 31.3$ & $R$ & 1400 & 1.410 & 1.804 & 33.5 & 240.5 & $237 \pm 4^{\circ}$ & $192 \pm 5^{\circ}$ \\
\hline 20151101 & $05: 28: 10.2$ & $R$ & 1400 & 1.560 & 1.806 & 33.3 & 237.8 & $233 \pm 3^{\circ}$ & $189 \pm 5^{\circ}$ \\
\hline 20151105 & $06: 11: 19.0$ & $R$ & 1400 & 1.587 & 1.802 & 33.3 & 238.3 & $222 \pm 6^{\circ}$ & $187 \pm 5^{\circ}$ \\
\hline 20151113 & 06:09:38.5 & $R$ & 1400 & 1.643 & 1.792 & 33.1 & 238.9 & $233 \pm 8^{\circ}$ & $176 \pm 6^{\circ}$ \\
\hline 20151115 & $05: 56: 26.4$ & $R$ & 1400 & 1.658 & 1.789 & 33.1 & 238.0 & $234 \pm 5^{\circ}$ & $177 \pm 5^{\circ}$ \\
\hline 20151122 & $05: 57: 59.0$ & $R$ & 1200 & 1.708 & 1.775 & 32.8 & 237.6 & $230 \pm 6^{\circ}$ & $172 \pm 5^{\circ}$ \\
\hline 20151203 & 05:55:06.2 & $R$ & 1400 & 1.790 & 1.745 & 32.3 & 238.1 & $231 \pm 3^{\circ}$ & $170 \pm 3^{\circ}$ \\
\hline 20151211 & 06:03:57.9 & $R$ & 1400 & 1.851 & 1.717 & 31.7 & 238.9 & $228 \pm 3^{\circ}$ & $169 \pm 4^{\circ}$ \\
\hline 20151215 & $06: 32: 46.2$ & $R$ & 1200 & 1.882 & 1.702 & 31.3 & 238.0 & $230 \pm 3^{\circ}$ & $167 \pm 3^{\circ}$ \\
\hline 20151224 & $05: 57: 01.8$ & $R$ & 1400 & 1.951 & 1.665 & 30.1 & 240.0 & $230 \pm 3^{\circ}$ & $162 \pm 4^{\circ}$ \\
\hline 20151231 & $05: 54: 20.2$ & $R$ & 1400 & 2.005 & 1.634 & 28.9 & 239.7 & $235 \pm 4^{\circ}$ & $160 \pm 3^{\circ}$ \\
\hline 20160104 & 06:39:11.1 & $R$ & 1400 & 2.036 & 1.616 & 28.1 & 241.2 & $238 \pm 3^{\circ}$ & $155 \pm 3^{\circ}$ \\
\hline 20160120 & $05: 34: 36.6$ & $R$ & 1400 & 2.159 & 1.546 & 24.0 & 239.9 & $239 \pm 3^{\circ}$ & $144 \pm 5^{\circ}$ \\
\hline 20160209 & $04: 15: 05.4$ & $R$ & 2000 & 2.312 & 1.489 & 16.4 & 238.4 & $240 \pm 3^{\circ}$ & $143 \pm 4^{\circ}$ \\
\hline 20160213 & 05:06:09.4 & $R$ & 1400 & 2.342 & 1.486 & 14.6 & 236.0 & $241 \pm 3^{\circ}$ & $140 \pm 3^{\circ}$ \\
\hline 20160217 & $03: 51: 31.5$ & $R$ & 1200 & 2.372 & 1.485 & 12.7 & 233.3 & $241 \pm 3^{\circ}$ & $140 \pm 3^{\circ}$ \\
\hline 20160225 & $02: 23: 44.6$ & $R$ & 1400 & 2.432 & 1.495 & 9.0 & 231.0 & $234 \pm 3^{\circ}$ & $144 \pm 3^{\circ}$ \\
\hline 20160309 & $02: 27: 31.3$ & $R$ & 1400 & 2.529 & 1.548 & 4.1 & 245.7 & $242 \pm 3^{\circ}$ & $148 \pm 3^{\circ}$ \\
\hline 20160312 & $23: 46: 04.0$ & $R$ & 800 & 2.558 & 1.573 & 3.8 & 262.8 & $240 \pm 6^{\circ}$ & $152 \pm 6^{\circ}$ \\
\hline 20160313 & $23: 47: 46.0$ & $R$ & 1200 & 2.573 & 1.588 & 3.8 & 266.4 & $243 \pm 4^{\circ}$ & $154 \pm 3^{\circ}$ \\
\hline 20160314 & $00: 44: 53.3$ & $R$ & 1200 & 2.573 & 1.588 & 3.9 & 266.4 & $244 \pm 5^{\circ}$ & $151 \pm 5^{\circ}$ \\
\hline 20160314 & $01: 41: 37.1$ & $R$ & 1200 & 2.573 & 1.588 & 3.9 & 266.4 & $242 \pm 4^{\circ}$ & $152 \pm 3^{\circ}$ \\
\hline 20160314 & $02: 39: 23.4$ & $R$ & 1200 & 2.573 & 1.588 & 3.9 & 266.4 & $244 \pm 3^{\circ}$ & $152 \pm 3^{\circ}$ \\
\hline 20160314 & $03: 35: 51.3$ & $R$ & 1200 & 2.573 & 1.588 & 3.9 & 266.5 & $242 \pm 3^{\circ}$ & $151 \pm 2^{\circ}$ \\
\hline 20160314 & $23: 29: 58.4$ & $R$ & 1200 & 2.580 & 1.596 & 3.9 & 266.5 & $247 \pm 4^{\circ}$ & $156 \pm 3^{\circ}$ \\
\hline 20160315 & $00: 37: 22.4$ & $R$ & 1200 & 2.580 & 1.596 & 4.0 & 269.3 & Contaminated & Contaminated \\
\hline 20160315 & $01: 36: 26.7$ & $R$ & 1200 & 2.580 & 1.596 & 4.0 & 269.3 & Contaminated & Contaminated \\
\hline 20160315 & $02: 35: 45.9$ & $R$ & 1200 & 2.580 & 1.596 & 4.0 & 269.3 & Contaminated & Contaminated \\
\hline 20160315 & $03: 34: 23.6$ & $R$ & 1200 & 2.580 & 1.596 & 4.0 & 269.3 & Contaminated & Contaminated \\
\hline 20160317 & 01:50:16.1 & $R$ & 1400 & 2.587 & 1.604 & 4.4 & 269.3 & $240 \pm 3^{\circ}$ & $152 \pm 4^{\circ}$ \\
\hline 20160320 & $01: 51: 53.0$ & $R$ & 1000 & 2.609 & 1.630 & 5.3 & 258.6 & $250 \pm 6^{\circ}$ & $150 \pm 5^{\circ}$ \\
\hline 20160423 & $23: 23: 38.3$ & $R$ & 1200 & 2.857 & 2.098 & 15.5 & 270.0 & $241 \pm 8^{\circ}$ & $163 \pm 4^{\circ}$ \\
\hline 20160523 & $22: 41: 09.2$ & $R$ & 1400 & 3.060 & 2.661 & 18.8 & 279.9 & $252 \pm 7^{\circ}$ & $186 \pm 5^{\circ}$ \\
\hline 20160527 & $23: 17: 46.8$ & $R$ & 1000 & 3.086 & 2.742 & 18.9 & 280.9 & $257 \pm 8^{\circ}$ & $188 \pm 6^{\circ}$ \\
\hline 20160601 & 23:09:33.1 & $R$ & 1000 & 3.119 & 2.843 & 18.9 & 282.0 & $252 \pm 8^{\circ}$ & $182 \pm 8^{\circ}$ \\
\hline 20160606 & $22: 41: 12.6$ & $R$ & 1400 & 3.151 & 2.945 & 18.8 & 283.1 & $247 \pm 12^{\circ}$ & Insufficient $\mathrm{S} / \mathrm{N}$ \\
\hline 20160615 & $23: 17: 10.0$ & $R$ & 1400 & 3.215 & 3.150 & 18.5 & 284.7 & $260 \pm 18^{\circ}$ & Insufficient $\mathrm{S} / \mathrm{N}$ \\
\hline 20160623 & $21: 47: 01.8$ & $R$ & 1400 & 3.260 & 3.313 & 17.8 & 285.9 & Insufficient $\mathrm{S} / \mathrm{N}$ & Insufficient $\mathrm{S} / \mathrm{N}$ \\
\hline 20160701 & $22: 40: 49.8$ & $R$ & 1400 & 3.315 & 3.474 & 17.1 & 287.0 & $238 \pm 20^{\circ}$ & Insufficient $\mathrm{S} / \mathrm{N}$ \\
\hline 20160713 & $22: 24: 32.5$ & $R$ & 1400 & 3.389 & 3.708 & 15.8 & 288.2 & Insufficient $\mathrm{S} / \mathrm{N}$ & Insufficient $\mathrm{S} / \mathrm{N}$ \\
\hline
\end{tabular}

Notes. The directions of the jets are presented as an angle between celestial north, the cometocenter, and the peak of the surface brightness associated with the jet at projected distance of $10^{4} \mathrm{~km}$. 
Table A.2. Successful observations of comet 67P/C-G in $V$-band with the Nordic Optical Telescope.

\begin{tabular}{|c|c|c|c|c|c|c|c|c|c|}
\hline $\begin{array}{c}\text { Date of } \\
\text { observation }\end{array}$ & UT & Filter & $\begin{array}{l}\text { Exposure } \\
\text { time (s) }\end{array}$ & $r$ & Delta & $\begin{array}{l}\text { Phase } \\
\text { angle }\end{array}$ & $\begin{array}{l}\text { Direction of } \\
\text { the Sun (deg) }\end{array}$ & $\begin{array}{c}\text { Subsolar jet } \\
\text { direction (deg) }\end{array}$ & $\begin{array}{c}\text { southern jet } \\
\text { direction (deg) }\end{array}$ \\
\hline 20150701 & $04: 29: 23.9$ & $V$ & 1000 & 1.355 & 1.923 & 30.5 & 278.4 & $210 \pm 6^{\circ}$ & Not visible \\
\hline 20150706 & $04: 25: 10.9$ & $V$ & 1200 & 1.332 & 1.893 & 31.1 & 275.9 & $203 \pm 8^{\circ}$ & Not visible \\
\hline 20150717 & $04: 12: 14.4$ & $V$ & 1000 & 1.290 & 1.839 & 32.4 & 270.1 & $216 \pm 6^{\circ}$ & Not visible \\
\hline 20150726 & $04: 05: 51.5$ & $V$ & 1000 & 1.265 & 1.807 & 33.1 & 265.3 & $229 \pm 5^{\circ}$ & Not visible \\
\hline 20150807 & 04:11:40.6 & $V$ & 1000 & 1.246 & 1.780 & 33.7 & 259.1 & $227 \pm 4^{\circ}$ & $152 \pm 8^{\circ}$ \\
\hline 20150815 & $04: 19: 36.8$ & $V$ & 400 & 1.243 & 1.771 & 33.9 & 255.2 & $233 \pm 4^{\circ}$ & $157 \pm 6^{\circ}$ \\
\hline 20150821 & $05: 25: 25.4$ & $V$ & 1000 & 1.246 & 1.768 & 34.0 & 252.5 & $234 \pm 4^{\circ}$ & $156 \pm 6^{\circ}$ \\
\hline 20150825 & $04: 27: 23.5$ & $V$ & 1200 & 1.252 & 1.768 & 34.0 & 250.8 & $236 \pm 3^{\circ}$ & $179 \pm 7^{\circ}$ \\
\hline 20150826 & $04: 21: 52.8$ & $V$ & 1200 & 1.252 & 1.768 & 34.0 & 250.4 & Contaminated & Contaminated \\
\hline 20150904 & 04:40:19.9 & $V$ & 1000 & 1.270 & 1.772 & 33.9 & 247.0 & $229 \pm 4^{\circ}$ & $180 \pm 6^{\circ}$ \\
\hline 20150909 & $05: 45: 29.3$ & $V$ & 800 & 1.283 & 1.776 & 33.8 & 245.4 & $231 \pm 4^{\circ}$ & $186 \pm 5^{\circ}$ \\
\hline 20150913 & $04: 18: 47.6$ & $V$ & 1200 & 1.296 & 1.780 & 33.8 & 244.2 & $228 \pm 5^{\circ}$ & $190 \pm 6^{\circ}$ \\
\hline 20151008 & $04: 47: 59.1$ & $V$ & 1200 & 1.410 & 1.804 & 33.5 & 240.5 & $224 \pm 5^{\circ}$ & $192 \pm 5^{\circ}$ \\
\hline 20151101 & $04: 52: 46.3$ & $V$ & 1200 & 1.560 & 1.806 & 33.3 & 237.8 & $220 \pm 8^{\circ}$ & $189 \pm 6^{\circ}$ \\
\hline 20151105 & $05: 36: 35.7$ & $V$ & 1200 & 1.587 & 1.802 & 33.3 & 238.3 & $221 \pm 5^{\circ}$ & $187 \pm 5^{\circ}$ \\
\hline 20151113 & $05: 33: 03.4$ & $V$ & 1200 & 1.643 & 1.792 & 33.1 & 239.0 & $225 \pm 4^{\circ}$ & $170 \pm 8^{\circ}$ \\
\hline 20151115 & $05: 12: 54.1$ & $V$ & 800 & 1.658 & 1.789 & 33.1 & 238.0 & $229 \pm 6^{\circ}$ & $176 \pm 6^{\circ}$ \\
\hline 20151203 & 05:20:53.9 & $V$ & 1200 & 1.790 & 1.745 & 32.3 & 238.1 & $225 \pm 4^{\circ}$ & $171 \pm 4^{\circ}$ \\
\hline 20151211 & $05: 27: 40.3$ & $V$ & 1200 & 1.851 & 1.717 & 31.7 & 240.4 & $226 \pm 5^{\circ}$ & $171 \pm 4^{\circ}$ \\
\hline 20151215 & $05: 59: 30.8$ & $V$ & 1200 & 1.882 & 1.702 & 31.3 & 239.2 & $228 \pm 5^{\circ}$ & $168 \pm 4^{\circ}$ \\
\hline 20151224 & $05: 23: 41.7$ & $V$ & 1200 & 1.951 & 1.665 & 30.1 & 240.0 & $232 \pm 4^{\circ}$ & $158 \pm 5^{\circ}$ \\
\hline 20151231 & $05: 20: 37.1$ & $V$ & 1200 & 2.005 & 1.634 & 28.9 & 239.7 & $234 \pm 4^{\circ}$ & $156 \pm 4^{\circ}$ \\
\hline 20160104 & 05:51:30.4 & $V$ & 1200 & 2.036 & 1.616 & 28.1 & 241.3 & $236 \pm 5^{\circ}$ & $154 \pm 5^{\circ}$ \\
\hline 20160120 & $04: 55: 40.6$ & $V$ & 1200 & 2.159 & 1.546 & 24.0 & 239.9 & $235 \pm 3^{\circ}$ & $148 \pm 4^{\circ}$ \\
\hline 20160209 & 03:41:50.3 & $V$ & 1200 & 2.312 & 1.489 & 16.4 & 238.3 & $241 \pm 4^{\circ}$ & $135 \pm 5^{\circ}$ \\
\hline 20160217 & $03: 16: 20.2$ & V & 1000 & 2.372 & 1.485 & 12.7 & 233.3 & $240 \pm 4^{\circ}$ & $136 \pm 4^{\circ}$ \\
\hline 20160225 & 01:43:06.6 & $V$ & 1200 & 2.432 & 1.495 & 9.0 & 231.0 & $237 \pm 8^{\circ}$ & $137 \pm 6^{\circ}$ \\
\hline 20160309 & $01: 57: 28.2$ & $V$ & 800 & 2.529 & 1.548 & 4.1 & 245.7 & $241 \pm 4^{\circ}$ & $142 \pm 5^{\circ}$ \\
\hline 20160312 & $23: 06: 17.4$ & $V$ & 1000 & 2.558 & 1.573 & 3.8 & 262.8 & $242 \pm 5^{\circ}$ & $151 \pm 5^{\circ}$ \\
\hline 20160313 & $23: 24: 57.0$ & $V$ & 1200 & 2.573 & 1.588 & 3.8 & 266.4 & $244 \pm 3^{\circ}$ & $152 \pm 3^{\circ}$ \\
\hline 20160314 & $00: 25: 47.1$ & $V$ & 1000 & 2.573 & 1.588 & 3.9 & 266.4 & $242 \pm 3^{\circ}$ & $153 \pm 4^{\circ}$ \\
\hline 20160314 & 01:18:46.4 & $V$ & 1400 & 2.573 & 1.588 & 3.9 & 266.4 & $242 \pm 3^{\circ}$ & $151 \pm 4^{\circ}$ \\
\hline 20160314 & $02: 16: 35.2$ & $V$ & 1200 & 2.573 & 1.588 & 3.9 & 266.4 & $242 \pm 3^{\circ}$ & $151 \pm 3^{\circ}$ \\
\hline 20160314 & 03:13:04.8 & $V$ & 1200 & 2.573 & 1.588 & 3.9 & 266.5 & $243 \pm 3^{\circ}$ & $151 \pm 3^{\circ}$ \\
\hline 20160314 & 23:07:09.3 & $V$ & 1200 & 2.580 & 1.596 & 3.9 & 266.5 & $245 \pm 4^{\circ}$ & $152 \pm 4^{\circ}$ \\
\hline 20160315 & $00: 14: 33.0$ & $V$ & 1200 & 2.580 & 1.596 & 4.0 & 266.6 & Contaminated & Contaminated \\
\hline 20160315 & $01: 13: 38.3$ & $V$ & 1200 & 2.580 & 1.596 & 4.0 & 269.3 & Contaminated & Contaminated \\
\hline 20160315 & $02: 12: 57.2$ & $V$ & 1200 & 2.580 & 1.596 & 4.0 & 266.4 & Contaminated & Contaminated \\
\hline 20160315 & 03:11:33.8 & $V$ & 1200 & 2.580 & 1.596 & 4.0 & 266.4 & Contaminated & Contaminated \\
\hline 20160317 & 01:17:05.8 & $V$ & 1000 & 2.587 & 1.604 & 4.4 & 266.6 & $244 \pm 6^{\circ}$ & $149 \pm 6^{\circ}$ \\
\hline 20160320 & $00: 46: 12.2$ & $V$ & 800 & 2.609 & 1.630 & 5.3 & 258.6 & $248 \pm 4^{\circ}$ & $149 \pm 5^{\circ}$ \\
\hline 20160523 & $22: 06: 42.8$ & $V$ & 1200 & 3.060 & 2.661 & 18.8 & 270.0 & Insufficient $\mathrm{S} / \mathrm{N}$ & Insufficient $\mathrm{S} / \mathrm{N}$ \\
\hline 20160527 & $22: 35: 59.7$ & $V$ & 1200 & 3.086 & 2.742 & 18.9 & 280.9 & $250 \pm 6^{\circ}$ & Insufficient $\mathrm{S} / \mathrm{N}$ \\
\hline 20160601 & $22: 27: 42.4$ & $V$ & 1200 & 3.119 & 2.843 & 18.9 & 282.0 & $246 \pm 10^{\circ}$ & Insufficient $\mathrm{S} / \mathrm{N}$ \\
\hline 20160606 & $22: 05: 35.3$ & $V$ & 1200 & 3.151 & 2.945 & 18.8 & 283.0 & $255 \pm 8^{\circ}$ & Insufficient $\mathrm{S} / \mathrm{N}$ \\
\hline 20160615 & $22: 40: 18.8$ & $V$ & 1200 & 3.215 & 3.150 & 18.5 & 284.7 & Insufficient $\mathrm{S} / \mathrm{N}$ & Insufficient $\mathrm{S} / \mathrm{N}$ \\
\hline 20160623 & $21: 13: 36.9$ & $V$ & 1200 & 3.260 & 3.313 & 17.8 & 286.0 & Insufficient $\mathrm{S} / \mathrm{N}$ & Insufficient $\mathrm{S} / \mathrm{N}$ \\
\hline 20160701 & $22: 05: 13.6$ & $V$ & 1200 & 3.315 & 3.474 & 17.1 & 287.0 & Insufficient $\mathrm{S} / \mathrm{N}$ & Insufficient $\mathrm{S} / \mathrm{N}$ \\
\hline
\end{tabular}

Notes. The directions of the jets are presented as an angle between celestial north, the cometocenter, and the peak of the surface brightness associated with the jet at projected distance of $10^{4} \mathrm{~km}$. 